\title{
Pattern Evaluation Proved To Be Important For Decision Support in Data Warehouse
}

\author{
Trivedi Prakashkumar Hitendrabhai ${ }^{1}$ Trivedi Jaydipkumar Hitendrabhai ${ }^{2}$ \\ ${ }^{I}$ Assistant professor, $G K$ \& C K Bosmia College,Jetpur,Gujarat,India \\ ${ }^{2}$ Assistant professor, Merchant College Of Management Studies And Research,Visnagar, Gujarat,India.
}

\begin{abstract}
It is the mobile sensor network or satellite that is observing and obtaining the data pattern. The study focuses on spatio-temporal data and events pattern for the data mining and for data warehouse. There is a repository or database where sensor, satellite inputs or data are collected for analysis. The depiction of satellite or mobile sensor and network leads new path for patterns, data mining and data warehouse. From the remote, the data obtained by using the mobile sensor, satellite so that data mining technique perform for finding desirable patterns. The study introduces moving point object data and algorithm as an advance research. The existing concept of moving point object based flock and leadership algorithm is exercising with uninterrupted data movement. It instructs that data moving in interrupted state. Present study focuses on an implementation of moving point object based Flock and Leadership algorithm. The algorithm is functioning with moving point object data but interrupted condition. The interrupted data produced abnormal result because the input data is captured in interrupted state.

From the inputs the study observes or collects spatial, temporal based elements or properties. There will be Spatial Temporal an event pattern. After obtaining data by using mobile sensor network or satellite network. The patterns are essential and useful which is desirable. The study instructing that patterns evaluation proved to be important for decision support in data warehouse. Further the study focuses on significance of pattern and its evaluation. There are events like earthquake, flood, desert events that are observed or obtained as an input of the work. They were abnormal in input stage but the data mining process has made data proper and obtained proper resulting data. On the basis of the selected pattern or data there can be a decision. The decision can be forecasting for earthquake, flood, or desert events. Finally the study observes the performance of pattern in all the stages of the study and has obtained the result of its importance and performance of the pattern for the decision support system in data warehouse.
\end{abstract}

Keywords- Pattern Evaluation, Data Pattern

\section{INTRODUCTION}

This research work is focused on pattern evaluation. Pattern obtained from the process of data mining carried out from a large pool of data organized in VLDB(very large database) or Data Warehouse. The Data Warehouse/VLDB is dynamically populated getting data pumped into it from satellite and wireless sensor network. A mined data can be used by the company or organization for decision purpose. The work sequence to study the existing algorithm provides patterns, the analysis of these patterns and identify the gape. The core work of this research is to extend the existing algorithm to acquired the further feature in the gap. The work is trying to provide a proposed pattern as well as introduced existing pattern for the references. ${ }^{[1]}$ The sensor network satellite network play important role for data capturing. The work introducing mobile sensor network or satellite network that capture the data. The captured data is to be sent through network and central hub. There will be data mining performing on obtained data. The obtained data is not in a proper condition or not in a proper shape. So the data mining technique can make the data proper. The pattern available with proposed algorithm will be utilized for moving point object data.

The work is providing pattern and also introduced pattern evaluation. The work is proving pattern evaluation is important for decision support in data warehouse. There will be KDD for their desirable information. Experts, managers are taking decision on the basis of obtained patterns and data or mined data. The pattern evaluation is a part and parcel of data mining. So it is important for making decision by data warehouse. Pattern evaluation instructs us about identification of patterns that is essential.

\section{LITERATURE REVIEW}

The literature survey undertaken for this research is centre around related work done by other researchers on moving point object based pattern and provide its algorithms and methods for considering. Here objects or data are moving in uninterrupted condition. The study needs the consideration of existing literature. As indicated motion object and identical pattern in spatial temporal data set of data mining are observed and explained in the efficient detection of motion pattern in spatial temporal data set. ${ }^{[2]}$ But the work instruct only 
detection of motion pattern in spatial temporal data set. The study focusing on moving point object based pattern and provide its algorithms and methods for considering. Here objects or data are moving in uninterrupted condition. In the work "Finding REMO-detecting relative motion patterns in geospatial lifeline" have analyzed that there is fundamental concept of moving point object data. ${ }^{[11]}$ It also instruct that they were available since the development of GPS and Radio transmitters. H.J.Miller and J.Han, editor, Geographic Data Mining And Knowledge Discovery. ${ }^{[12]}$ Kay Romar,Distributed Mining of spatio-temporal event pattern in sensor network. ${ }^{[23]}$ The study based on sensor and provide analysis. There is data mining technique to discover frequent event pattern.JiaweiHan, Guozhu Dong, Yiwen Yin, Efficient Mining of Partial Periodic Pattern in Time series database have presented the periodic patterns. Time factors prove to be important in this work. The work also provides algorithams for efficient mining of partial periodic patterns ${ }^{[24]}$.Linghuan Hu, David Evan, Localization for Mobile Sensor Networks indicate the localization schemes for sensor networks for a nodes. In the same work several localization technique have explained. There is an instruction of location awareness for wireless sensor network in many application like environment monitoring. ${ }^{[25]}$ Guang Song, Yaoxin Zhon, Fei Ding and Song, A Mobile Sensor Network System For Monitoring Unfriendly Environments ${ }^{25]}$

\section{Analysis of An Existing Architecture.}

\section{ARCHITECTURE}

There is a kind of existing architectures indicated in Building The Data warehouse, ${ }^{[26]}$ which is using for decision support in the context of data mining and data warehouse. In this architecture instructs competitive advantage by presenting relevant information to measure performance and make judgment in order to win the competitive market space.In the same literature four different views of the data warehouse must be considered (1) The Top-Down View (2) The Data Source View (3) The Data warehouse View (4) The Business Query View

(1)The Top-Down View is focusing the current and forth coming need of the business.

(2) The Data Source View is instructing that the data is captured, stored and managed by data warehouse system. The view presents the individual source table to integrated source tables information may be detailed at various level of accuracy. In the same view instructing CASE tools or entity relationship model hold traditional data source.

(3) The Data warehouse view presents fact tables dimensions tables. Which represents the information that is stored within the data warehouse.

(4) The Business Query View is the result of data in the data warehouse based on the view of the user.

The existing warehouse system using Top-down Approach or Bottom-up approach. The existing system using data mining steps. 1 The removal of noise and inconsistent data functioning as data cleaning. 2 The combination of multiple source functioning as Data Integration.3 The data relevant for analysis is retrieved from database functioning as Data selection.4 The consolidation and transformation of data into forms appropriate for mining by using aggregation or summary of data functioning as Data Transformation.5 The use of intelligent methods to extract pattern from data functioning as Data Mining. 6 Identification of patterns that are interesting functioning as Pattern Evaluation.7Visualization technique are presented.

\section{Problem with Architecture.}

The data mining and data warehouse architecture is proper but the work functioning with 1.Sensor data detection, 2.Satellite data detection , 3. Data mining ,4. Data warehouse so it need combined architecture of the whole process. The study introduce a proposed architecture for obtain an advance patterns and data. It introduces the proposed and combined functionality based architecture. The architecture instructing detection of data, data mining and data warehousing for normal and abnormal, proper and improper data for providing proper shape or proper result of the data.

\section{Proposed Architecture.}

The study finding generates a proposed architecture for obtaining advanced patterns and data. The sensor or satellite based detection of data using flock and leadership's moving points and interrupted and uninterrupted data. The detected data can be in proper condition or can not be in a proper condition. So the normal as well as abnormal detected data need a reformation for proper shape or proper result. For reformation of the data there is a need of data mining technique which makes the data proper. At the end of the work provide decision or result using data warehouse. Thus all the functions like 1. Detection of data, 2. Reformation of data,3.Providing a decision need depiction of combined functionality. Which leads towards a development of an architecture. 


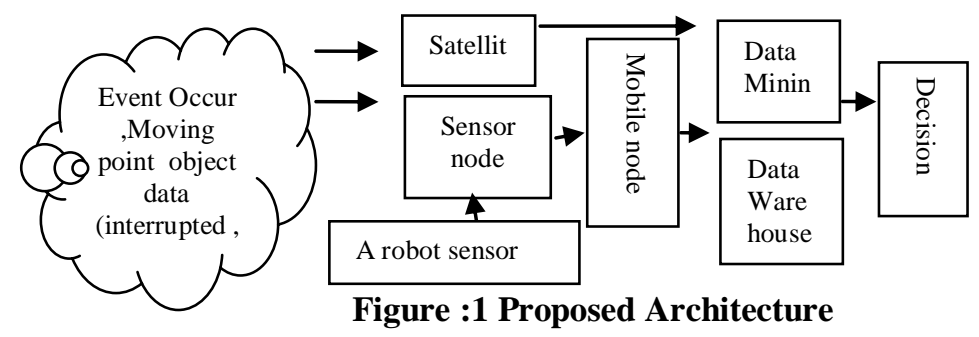

A combined architecture for Mobile sensor, Data mining and Data Warehouse. There are events obtaining as input of data from sensor node indicated in the figure. "Interrupted ", but in a moving conditioned object data as a Flock and Leadership format. Mobile node is collecting "obtained data" from sensor node. At the Data mining phase there is a process for data mining and observe the process of mining occur. A process contain Data

cleaning, Data Integration, Data Selection, Data Transformation, Data Mining, Pattern Evaluation and knowledge presentation. Here the work obtaining Spatial-Temporal and web based data from different sensor node and mobile nodes. There is KDD as desirable pattern. At the end mined data go for data warehouse for the further process.

\section{Justification of proposed Architecture.}

The justification of proposed architecture comprise under mention criteria. The goal of an Architecture for the research work is to enhance coordination, simplify integration, build a consistent infrastructure, and generally allow greater efficiencies in the development of detections of data, patterns, data mining and warehouse based decision and provide solutions. The intent of an Architecture program is to realize these goals while ensuring effective use of resources, thereby enabling consistent, effective delivery of data and patterns to the receiving end. An Architecture defines the roles, functionality, core work of flock and leadership format data, pattern, sensor detection of data, satellite detection of data, data mining for formation of interrupted and uninterrupted data, warehousing and decision-making criteria for the acquisition of proper pattern and results.

\section{Proposed Architecture Framework}

The Architecture is a data detection and pattern development driven by the needs of user ,decision makers, higher authority, manger and is founded on principles, best practices, trends, as well as Architecture Processes. The Architecture comprise in to four parts.1.Data detection by sensor network and satellite.2.Process using algorithms 3.Data mining and data warehouse 4.Decision

\section{Need For Pattern Evaluation.}

\section{PATTERN EVALUATION}

The goal of depiction of architecture is to instruct a pattern using technology. The workflow leads towards pattern evaluation. The out put of the data or shape of the data obtained by sensor node or satellite remains proper as well as improper and obtained as interrupted and uninterrupted condition so it need pattern evaluation. The evaluation of pattern need architecture based system for the further development.

\section{Pattern Evaluation And It's Effect}

Proposed Architecture depicted sensor network and mobile sensor network, ${ }^{[10]}$ for detecting data, observing, analyzing certain event of the real world. At the beginning the data or inputs are in abnormal condition. The streams of mobile rows sensor reading from every nodes are delivered to a central sink for further analysis. The work is finding the desirable patterns in the data resulting in a large volume that has to be delivered through the network. Now the study is experiencing mobile sensor network. There are subtle realworld influences and large scale are the cause of numerous bugs and indeterminist behavior of mobile sensor network. Again, since the nature of these problems is often unknown in advance and deployment support networks have been proposed to deliver high volume event logs from every mobile sensor node from ever mobile sensor node to a central sink for off-line analysis in order to identify patterns that lead to failure.

There would be data mining, where a user provide certain hints to the system on what kind of patterns to look for. The user must define a limited set of events which are potential interest. In earth quake monitoring application, these event might be defined by the origin of earth quake shocks, sound, ways earth movement in the sea area. The system will then discover frequent spatio-temporal and web based pattern among these events.

\section{Proposed Architecture Based System For Pattern Evaluation :}

Architecture based system for pattern evaluation undergo below mention process. There are certain rules and protocols that is essential for the process. The working Nodes are working on a particular time, the 
working node have their environment, surrounding or places. There will be time duration that will be regarded as a measurements. There will be definite condition that will affect on whole process or on whole system. There is time and some measurement for distance.

There will be certain study based on Sensor network. Environmental sensors are developed and used for environment assessment such as electromagnetic field, thermal and air environments. There will be some specified items for thermal environmental assessment are temperature, humidity.

\section{Using Mobile Sensor Network, Mining Of Spatio-Temporal Based Event Patterns:}

Using mobile sensor network, mining of spatio-temporal and web based event Patterns: subject undergo below mention area.

\section{System Overview For Mobile Sensor Network.}

There are many group of mobile sensor nodes. There will be base station and communication network and also client. Each sensor node collecting data and routing data peer to peer to base station. It not only has all capabilities of the static sensor nodes but also realize mobility by adding a robotic base and driver board. A base station is used to bridge the sensor network to another network such as internet. It is well suited for distributed measurement and control application. It's architecture divided into three different layers. (1) node layer: It consist of all the server nodes that will be static or mobile. This layer is directly enabled into physical world to get any kind of data. (2) Server layer: It includes server or server software.(3) The client layer: Client layer includes local client and remote client. The device of the client layer can be any smart terminals such as PC, PDA., Pocket PC, and smart phone.

\section{Process of Data Mining And Data Warehouse.}

Mobile node is gathering obtained data. At the mining phase there is a process for data mining. A process contain Data cleaning, Data Integration, Data Selection, Data Transformation, Data Mining, Pattern Evaluation and knowledge presentation. Here is the obtaining Spatial-Temporal and web based data from different sensor node and mobile nodes. At the end mined data go for data warehouse .

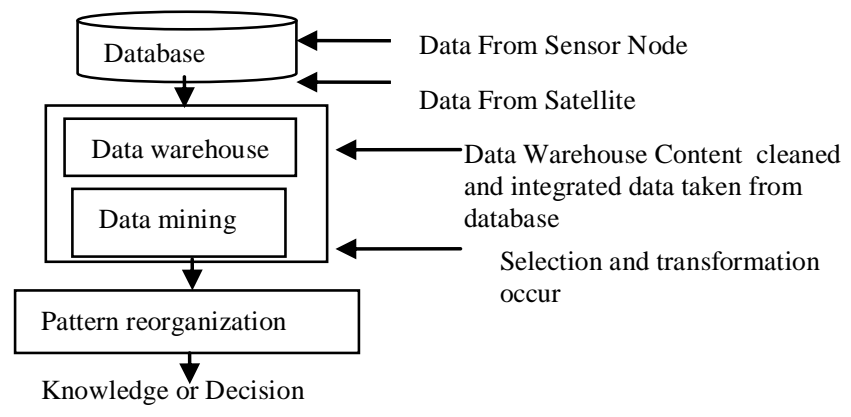

Figure 2 Process of data mining and data warehouse

\section{Indication of Motion Patterns In Spatio-Temporal Data Set.}

The architecture is using the algorithm for detection of data from the world using mobile sensor or satellite. The moving point data can be constant moving and not constant moving. Flock pattern indicated only constant moving data. It is the question that what about the data which is not constant moving or moving but in interrupted condition?

\section{Causes of Interruption:}

1.Not properly transmitted data.2.Not properly detected data.

3.Environmental obstacles.4.Poor result of multimedia camera for detecting data.

\section{Implementation Of Algorithm :}

Here the study focusing on moving point object data that can be analyzed through the discovery of patterns. As defined by Laubeet al. 2004, the computational efficiency of detecting from such spatio-temporal pattern, namely flock, leadership, convergence and encounter. According to Laubeet al these patterns are large enough subgroup of the moving point objects that exhibit similar movement in the sense of direction, heading for the same location ,and /or proximity. The work is analyzing a kind of object or data that is in a "Interrupted “, but in a moving condition .Moving point but in a interrupted conditioned object data can be analyzed through the discovery of patterns. By the use of techniques from computational statistical, including to detect these patterns. 


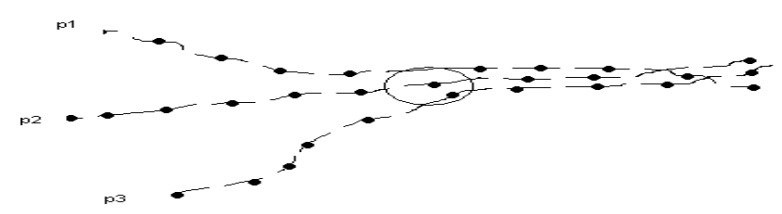

Figure : 3 " Interrupted " , but in a moving conditioned

object data as a Flock and Leadership format.

For estimating algorithms "Interrupted", but in a moving conditioned object data, the work will use the Flock and Leadership algorithms for the initial construction. As indicated by Laube et al .2004, Flock Parameters: $m>1$ and $r>0$. At least $m$ entities are within a circular region of radius $r$ and they move in the same direction. Leadership Parameters: $\mathrm{m}>1, \mathrm{r}>0$, and $\tau>0$. At least $\mathrm{m}$ entities are within a circular region of radius $r$, they move in the same direction, and at least one of the entities was already heading in this direction for at least $\tau$ time steps.

\section{Step For Current Algorithm :}

Step1. Take set of ' $\mathrm{n}$ ' moving data, minimize size $\mathrm{m}<=\mathrm{n}$

for subset to form a pattern.

Step2. The ' $n$ ' moving data's radius is ' $r$ '.

Step3. Separate the input data into 8 subsets According to their motion direction.

Step4. Take each subset separately.

\section{Result For Data Mining And Ware house:}

Data obtained by using "Interrupted", but in a moving conditioned object data as a Flock and Leadership format is not providing perfect result. The reason of interrupted data are 1 . Not properly transmitted data. 2. Not properly detected data. 3. Environmental obstacles. 4. Poor result of multimedia camera for detecting data. The data mining technique can regenerate the data as complete data or perfect data.

\section{Event Occurrence, Obtaining Data and Recognize Of An advance And Different Pattern.}

Here the study is depicting certain areas where the events occur. Earth quake occur at sea area Example. Arbi Sea or Kutch's Desert near the state of Gujarat in India. The event occurrence based on data. That the work is obtaining data from different sources like web sites, media like T.V radio, News Paper.

Event 1 : Earth quake occur at sea area Example. Arbi Sea or

Kutch's Desert near the state of Gujarat in India.

Pattern 1: Motion Patterns in Spatio-Temporal Data Sets

Pattern 2: "Interrupted ", but in a moving conditioned object

data as a Flock and Leadership format based pattern.

The moving point object data pattern ("Interrupted".),Thus the work have point data pattern, Points may be used to indicate spatial occurrences or events and their spatial pattern. Point pattern analysis is concerned with the location of events and with answering questions about the distribution of those locations, whether they are clustered, distributed randomly. Point pattern analysis is used to identify whether occurrences or events are interrelated or not. The main characteristic of the point data pattern is its central tendency. The central tendency of a set of values gives some indication of the average value as their representation. When dealing with spatial data set, the concept of average in classical statistics can be extended to the concept of center, as a measure of spatial central tendency.

\section{Decision Obtained Due To Only And Only Based On Pattern}

The statistical operations for central tendency of the distribution for the data of earthquake epicenters in and around Gujarat were done with the help of Arc View 3.2 GIS software. The spatial mean center for the distribution of the epicenters were calculated. Both spatial mean and the median center when calculated with weightage given to the magnitude of the earthquake for that particular epicenter did not show much variance in their locations, thereby indicating that the epicenters with contrasting magnitude i.e. very low or very high compared to the magnitude of the majority of the earthquake epicenters do not lie scattered at far distant places. It shows that the entire area is continuously being struck by earthquakes of varying magnitude that seem to be controlled by the same structure.

Pattern Evaluation Proved To Be Important for Decision In Data warehouse.

A data warehouse provide competitive advantage by presenting relevant information from which to measure performance and make critical judgment. Here data mining supports knowledge discovery by finding 
hidden patterns like Spatio-Temporal Data Set. The work is functioning on moving point object data that can be analyzed through the discovery of patterns. It utilized Flock and Leadership algorithms for the initial construction. Here the study introducing a "Interrupted, but in moving conditioned object data" algorithm. A data warehouse is storing data as a big repository in support of management's decision making process. It forecasts or demonstrate environmental matter. It focus on modeling and analysis of data for decision-makers. As practical approach the work have obtained pattern for earth quake from Gujarat a state of India. A data warehouse have used that pattern and provided environmental decision.

\section{Characteristics of moving point object data}

The study has estimated Interrupted and Uninterrupted moving point object data pattern. That is moving in direction as indicated flock and leadership algorithms. It instructs a kind of object or data that is in an "Interrupted ", but in a moving condition. Moving point but in a interrupted conditioned object data can be analyzed through the discovery of patterns.

\section{Characteristics of point data pattern}

Points may be used to indicate spatial occurrences or events and their spatial pattern. Point pattern analysis is concerned with the location of events and with answering questions about the distribution of those locations, whether they are clustered, distributed regularly. Point pattern analysis is used to identify whether occurrences or events are interrelated or not. The main characteristic of the point data pattern is its central tendency. The central tendency of a set of values gives some indication of the average value as their representation. When dealing with spatial data set, the concept of average in classical statistics can be extended to the concept of center, as a measure of spatial central tendency. The earthquake's epicenter was located about $80 \mathrm{~km}$ east of the city of Bhuj, Gujarat, but the MISR instrument found dewatering, or release of water and sediment due to compression and liquefaction, as far as $200 \mathrm{~km}$ from the epicenter. Additionally, there was significant dewatering all along an 80-100 kms wide ancient salt lake bed to the north of Bhuj, known as the Rann of Kutch. In the days to weeks following the earthquake, along with ground cracks and other types of deformation, water flowed to the surface and progressively evaporated in various places. A year later, scientists could still observe the consequences of the earthquake across the Rann because the water that came up to the surface was very salty. After evaporation, the salt was left on the ground and MISR was able to detect it also. The MISR instrument views the sunlit face of the Earth simultaneously at nine widely spaced angles, and provides ongoing global coverage with high spatial detail. Its imagery is carefully calibrated to provide accurate measurements of the brightness, contrast, and color of reflected sunlight. One way MISR registers surface features is by picking up different wavelengths of light as they are reflected off the Earth's surface. As the satellite passes overhead, MISR collects information over a $400 \mathrm{~km}$ swath at a spatial resolution of 275 meters, instantaneously assessing surface features over large regions. Since the bright soils of the Rann of Kutch reflect most of the Sun's incoming near-infrared radiation, and water bodies absorb near-infrared radiation, MISR can detect the contrast and thereby tell where dewatering from the earthquake occurred. Changes in reflection at different view angles also proved advantageous to identify the presence of surface water in other regions. In the Gujarat region showing locations of documented liquefaction features relative to the epicenter of the January 26 , 2001 earthquake details. Changes in reflection at different view angles the identification of surface water. viewing (nadir) camera are displayed as green. Surface water in the Rann of Kutch and along the Nagar-Parkar fault near the Indo-Pakistan border. Each image covers an area of $215 \mathrm{~km} \mathrm{x} 156 \mathrm{~km}$. Credit: NASA/GSFC/LaRC/JPL, MISR Team .

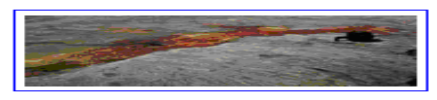

Fig .4 Sand Blow Deposit in Great Rann

It shows a sand blow deposit. When waves from a large earthquake pass through wet, loose sand, patches of sand erupt onto the ground and form sand blow deposits. This image shows a sand blow deposit that formed in the Great Rann about $20 \mathrm{~km}$. A month after the earthquake, the sand blow and surrounding area were still wet, salt had begun to precipitate, and iron-oxides had formed in vent area. Credit: M. Tuttle.

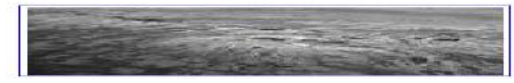

Fig 5 Sand Blow In Mud Flats

This sand blow formed in mud flats near Kandla about $50 \mathrm{~km}$.

\section{RESULT}

- The research work instructs that the pattern evaluation is important for decision in data warehouse.

- The study indicates moving point object data in interrupted condition.

- A pattern is important for data mining and for data warehouse. 
- Sensor node, satellite obtaining interrupted, uninterrupted data.

- Using data mining and data warehouse the interrupted and uninterrupted data is shaping for a resulting data. Flock but interrupted condition pattern based data moving.

\begin{tabular}{|l|l|l|}
\hline No & Pattern & Description \\
\hline 1 & $\begin{array}{l}\text { Flock } \\
\text { Parameters: }\end{array}$ & $\begin{array}{l}\mathrm{m}>1 \text { and } \mathrm{r}>1 \text { at least m entitles } \\
\text { is within a circular region of } \\
\text { radius } \mathrm{r} \text { and move in the same } \\
\text { direction in un interrupted } \\
\text { condition. }\end{array}$ \\
\hline 2 & $\begin{array}{l}\text { Interrupted } \\
\text { condition } \\
\text { object } \\
\text { parameters : }\end{array}$ & $\begin{array}{l}\mathrm{m}>1 \text { and } \mathrm{r}>1 \text { at least m entitles } \\
\text { is within a circular region of } \\
\text { radius } \mathrm{r} \text { and move in the same } \\
\text { direction in interrupted } \\
\text { condition }\end{array}$ \\
\hline
\end{tabular}

There are below mention patterns

\begin{tabular}{|l|l|l|}
\hline $\begin{array}{l}\text { N } \\
\mathrm{o}\end{array}$ & $\begin{array}{l}\text { Moving point object } \\
\text { data at one side in } \\
\text { interrupted condition }\end{array}$ & $\begin{array}{l}\text { Moving point object data at } \\
\text { another side And role of data } \\
\text { mining and data warehouse }\end{array}$ \\
\hline 1 & $\begin{array}{l}\text { The real data Sand } \\
\text { blow in mud flats } \\
\text { At time 1: }\end{array}$ & $\begin{array}{l}\text { The resulting data } \\
\text { Ahe average result of moving } \\
\text { point Object data }\end{array}$ \\
\hline & At time 2: & \\
\hline
\end{tabular}

\begin{tabular}{|l|l|l|}
\hline 2 & At Time 1 & \\
& Colorodo River: & \\
\hline & At Time 2 & Resulting Figure \\
& Colorodo River & \\
\hline & At Time 3 & \\
\hline
\end{tabular}

\begin{tabular}{|c|c|c|c|}
\hline $\begin{array}{l}\text { Input Data } \\
\text { Condition }\end{array}$ & $\begin{array}{l}\text { Data } \\
\text { Detection } \\
\text { Agent }\end{array}$ & $\begin{array}{l}\text { Using Data MiningAnd } \\
\text { Data warehouse, Result } \\
\text { obtaining After perfoRming } \\
\text { Pattern ev.syst. }\end{array}$ & $\begin{array}{l}\text { Proof After Perfor-ming } \\
\text { Pattern Evaluation } \\
\text { System. Information }\end{array}$ \\
\hline $\begin{array}{l}\text { 1.Uninterrupted } \\
\text { condition at the } \\
\text { beginning. }\end{array}$ & $\begin{array}{l}\text { Satellite data for sand } \\
\text { blow deposit in great rann }\end{array}$ & Proper Result Of Pattern & $\begin{array}{l}\text { Sand Blow Deposit } \\
\text { inGreatRann }\end{array}$ \\
\hline $\begin{array}{l}\text { 2.Uninterrupted at } \\
\text { the beginning. } \\
\qquad \text { ISSN: } 2250-3021\end{array}$ & $\begin{array}{l}\text { Sensor node data taken by } \\
\text { 18,Type acc,Direction :H } \\
\text { osition }(\mathrm{x}, \mathrm{y}, \mathrm{z}): 822395206\end{array}$ & $\begin{array}{l}\text { Proper } \\
\text { Result } \\
\text { Of } \\
\text { P.entign.org }\end{array}$ & $\begin{array}{l}\text { Sensor infer - } \mathrm{P} \text { a g e } \\
\text { mation }\end{array}$ \\
\hline
\end{tabular}


Pattern Evaluation Proved To Be Important For Decision Support in Data Warehouse

\begin{tabular}{|l|l|l|l|}
\hline $\begin{array}{l}\text { 3.Interrup- ted at the } \\
\text { beginning. }\end{array}$ & $\begin{array}{l}\text { Satellite data for send in } \\
\text { mud. }\end{array}$ & Proper Result of Pattern & $\begin{array}{l}\text { Send blow } \\
\text { in mud }\end{array}$ \\
\hline $\begin{array}{l}\text { 4.Interrup- } \\
\text { ted at the beginning. }\end{array}$ & $\begin{array}{l}\text { Sensor } \\
\text { node data } \\
\text { Colorode river }\end{array}$ & $\begin{array}{l}\text { Proper } \\
\text { Result } \\
\text { of Pattern }\end{array}$ & $\begin{array}{l}\text { Colorodo } \\
\text { River }\end{array}$ \\
\hline $\begin{array}{l}\text { half interup } \\
\text { ted Halfunin } \\
\text { terrupted at begin. }\end{array}$ & $\begin{array}{l}\text { Satellite } \\
\text { for } \\
\text { moon }\end{array}$ & $\begin{array}{l}\text { Proper } \\
\text { Result } \\
\text { Of Pattern }\end{array}$ & Moon \\
\hline
\end{tabular}

\section{CONCLUSSION}

The research work depicted as Introduction, Literature review Analysis of existing Architecture and need for improvement. Proposed architecture and Concept of Computer Science, Pattern evaluation and its effects and data Capturing With Prototype for implementation of the research.

\section{REFERENCES}

[1] S. Arya, D. M. Mount, N. S. Netanyahu, R. Silverman, and A. Y. Wu. An optimal algorithm for approximate nearest neighbor searching fixed dimensions. Journal of the ACM, 45(6):891-923, 1998.

[2] B. Chazelle. Cutting hyperplanes for divide-andconquer. Discrete and Computational Geometry, 9(2):145-158, 1993.

[3] T. H. Cormen, C. E. Leiserson, R. L. Rivest, and C. Stein. Introduction to Algorithms. MIT Press,ambridge, MA, 2 nd edition, 2001.

[4] J. Erickson. New lower bounds for convex hull problems in odd dimensions. SIAM Journal of Computing, 28:1198-1214, 1999.

[5] A.U. Frank, J.F. Raper, and J.-P. Cheylan, editors. Life and motion of spatial socio-economic units. Taylor \& Francis, London, 2001.

[6] L. J. Guibas, M. H. Overmars, and J.-M. Robert. The exact fitting problem for points. Computational Geometry - Theory and Applications, 6:215-230, 1996.

[7] D. Halperin. Arrangements. In J.E. Goodman and J. O’Rourke, editors, Handbook of Discrete and Computational Geometry, chapter 24, pages 529-562. Chapman \& Hall/CRC, Boca Raton, 2nd edition, 2004.

[8] S. Iwase and H. Saito, Tracking soccer player using multiple views. In Proc. IAPR Workshop on Machine Vision Applications (MVA02), 2002.

[9] G. Kollios, S. Sclaroff, and M. Betke. Motion mining:discovering spatio-temporal patterns in databases of human motion. In Proc. ACM SIGMOD Workshop on Research Issues in Data Mining and Knowledge Discovery, 2001.

[10] P. Laube and S. Imfeld. Analyzing relative motion within groups of trackable moving point objects. In GIScience 2002, number 2478 in Lect. Notes in Computer Science, pages 132-144, Berlin, 2002. Springer.

[11] P. Laube, M. van Kreveld, and S. Imfeld. Finding REMO - detecting relative motion patterns in geospatial lifelines. In Proc. 11th Int. Symp. on Spatial Data Handling, 2004.

[12] H.J. Miller and J. Han, editors. Geographic Data Mining and Knowledge Discovery. Taylor \& Francis, London,2001.

[13] K. Mulmuley. Computational Geometry: An Introduction Through Randomized Algorithms. Prentice Hall, Englewood Cliffs, NJ, 1994.

[14] T. Ott and F. Swiaczny. Time-Integrative Geographic Information Systems. Springer, Berlin, 2001.

[15] Porcupine caribou herd satellite collar project. http://www.taiga.net/satellite/.

[16] J. Roddick, K. Hornsby, and M. Spiliopoulou. An updated bibliography of temporal, spatial, and spatiotemporal data mining research. In TSDM 2000, number 2007 in Lect. Notes in Artificial Intelligence, pages 147-163, Berlin, 2001. Springer.

[17] H. Samet. The design and analysis of spatial data structures. Addison-Wesley, 1990.[18] C.-B. Shim and Jae-Woo Chang. A new similar trajectory retrieval scheme using k-warping distance algorithm for moving objects. In Proc. 4th Int. Conf. on Advances in Web-Age Information Management, (WAIM 2003), number 2762 in Lect. Notes in Computer Science,pages 433-444, 2003.

[19] N. Sumpter and A.J. Bulpitt. Learning spatio-temporal patterns for predicting object behaviour. Image Vision Comput., 18:697-704, 2000.

[20] G. Fejes T'oth. Packing and covering. In J.E. Goodman and J. O'Rourke, editors, Handbook of Discrete and Computational Geometry, chapter 2, pages 25-52. Chapman \& Hall/CRC, Boca Raton, 2nd edition, 2004.

[21] M. Vlachos, G. Kollios, and D. Gunopulos. Discovering similar multidimensional trajectories. In Proc. 18th Int. Conf. on Data Engineering (ICDE’02), 2002.

[22] Joachim Gudmundsson, Marc van Kreveld Bettin Speckmann, efficient detection of motion pattern in spatial temporal data set.

[23] Kay Romar,Distributed Mining of spatio-temporal event pattern in sensor network.

[24] JiaweiHan, Guozhu Dong, Yiwen Yin, Efficient Mining of Partial Periodic Pattern in Time series database have presented the periodic Patterns.

[25] Linghuan Hu, David Evan, Localization for Mobile Sensor Networks indicate the localization schemes for sensor networks for a nodes.

[26] W. H. Inmon, Building The Data warehouse 